\title{
Effect of Running Environment and Slope Gradient on Lower Limb Muscle Activation
}

\author{
Theodoros Roussos ${ }^{1, ~ *}$, Athanasia Smirniotou ${ }^{2}$, Anastasios Philippou ${ }^{3}$, Antonis Galanos ${ }^{1}$, \\ Ioannis Triantafyllopoulos ${ }^{1}$ \\ ${ }^{1}$ Laboratory for the Research of Musculoskeletal System, Medical School, National and Kapodistrian University of Athens, Athens, Greece \\ ${ }^{2}$ School of Physical Education and Sport Science, National and Kapodistrian University of Athens, Athens, Greece \\ ${ }^{3}$ Department of Physiology, Medical School, National and Kapodistrian University of Athens, Athens, Greece
}

\section{Email address:}

theodoros.roussos@yahoo.gr (T. Roussos), asmirn@phed.uoa.gr (A. Smirniotou), tfilipou@med.uoa.gr (A. Philippou), galanostat@otenet.gr (A. Galanos), sportdoc@otenet.gr (I. Triantafyllopoulos)

${ }^{*}$ Corresponding author

\section{To cite this article:}

Theodoros Roussos, Athanasia Smirniotou, Anastasios Philippou, Antonis Galanos, Ioannis Triantafyllopoulos. Effect of Running Environment and Slope Gradient on Lower Limb Muscle Activation. American Journal of Sports Science. Vol. 7, No. 1, 2019 , pp. $20-25$. doi: $10.11648 /$ j.ajss.20190701.14

Received: February 23, 2019; Accepted: March 30, 2019; Published: April 18, 2019

\begin{abstract}
The purpose of this study was to assess whether the electromyographic (EMG) activity of the lower limb muscles - rectus femoris, vastus medialis, bicep femoris and gastrocnemius - during running is affected by different running environments and varied slope gradients. Data collected from this study would be useful for specific training programs adopted from different style runners. Ten male recreational runners participated in the current study. Each of the ten participants, was assessed in two different running conditions, treadmill running and over-ground running, which was consisted of a $20 \mathrm{~m}$ distance running at a velocity of $11 \pm 1 \mathrm{~km} / \mathrm{h}$ at each of the following slope gradients $-8 \%,-4 \%, 0 \%, 4 \%, 8 \%-$ in a randomized order. Findings revealed that when over-ground running EMG activity of the lower limb muscles was $16.54 \%$ higher compared to treadmill running. Treadmill running is characterized by reduced metabolic cost, which is related to lower muscle activation requirements. Varied slope gradients found to affect muscle activation. During uphill running, lower limb muscle activation was significantly higher compared to level and downhill running, independently of running environment. However, running at negative slope gradients was associated with significantly greater muscle activation compared to level running, only on the treadmill condition.
\end{abstract}

Keywords: Electromyographic Activity, Overground Running, Treadmill Running, Slope Gradient

\section{Introduction}

The physical activity of running is a key element in most conditioning programs. Running at different slope gradients (positive and negative) and varied environments (overground and treadmill running) is a commonly used method in training programs for distance runners.

Inclined support surfaces affect the control of movement in terms of the maintenance of an upright posture, the foot strike patterns and the related centre of pressure in anteriorposterior direction during stance, and the corresponding muscle activity. Running at inclined surfaces influences kinematic and kinetic characteristics of running technique and lower limb joints function. Downhill running is characterized by greater knee flexion angle [1] which is associated with an increased vertical displacement of the centre of mass $[2,3]$. Uphill running is characterized by a reduction in vertical displacement of the centre of mass with a simultaneous decrease in maximal vertical force components, compared to level and downhill running. At more pronounced slopes ( $\pm 5 \%$ and $\pm 8 \%$ ) maximal vertical force was lower when running uphill than downhill. Higher gradients were also reported to be associated with a higher step frequency during uphill running [1,3-6]. The proportion of contact time during the step cycle was grater at increased gradients (from $+3 \%$ to $+11 \%$ ) $[3,4,7]$, while flight time and 
step length were decreased $[4,5]$.

These changes in spatiotemporal parameters caused by varied gradients interact with the work performed by muscle groups during running. However, there is little information on how lower limb muscles activity is affected by positive and negative slope gradients. Uphill running is characterized by increased demands for muscle mechanical work in order to increase the body potential energy. It has been referred that the most of the work necessary to perform uphill running is produced at the hip joint, while the knee and ankle joints perform similar functions at all gradients $\left(0^{\circ}, 6^{\circ}, 1^{\circ}\right)$. Mechanical work produced at the hip joint increased significantly with increasing slope gradient, as a result of either an increase in the moment of muscle force developed by hip extensors or through power transfer by knee extensors to the hip via the hamstrings [8]. Greater activation of the muscle groups surrounding the hip joint (hamstrings and iliopsoas) and the muscles surrounding the knee joint (vastii muscles and rectus femoris) was observed during uphill running at a high speed $(5 \mathrm{~m} / \mathrm{sec})$, compared to level running [9].

The work performed by muscle groups is partially affected by the foot strike pattern adopted during locomotion [10-12]. During level and downhill running, anterior patterns (midfoot strike and forefoot strike patterns) are associated with greater plantar flexion and knee flexion and with higher gastrocnemius lateralis and bicep femoris activity and lower tibialis anterior and vastus lateralis activity, compared to posterior patterns (rear foot strike) [10-14].

During dynamic locomotion - walking and running muscular function is also affected by running environments. When running on a treadmill, the supporting ground (treadmill belt) is moving relatively to subjects' centre of mass, which is opposite to real world bipedal locomotion where subjects' centre of mass moves relatively to the supporting ground [15]. When comparing over-ground and treadmill running, differences concerning the kinematic and kinetic parameters have been observed. Treadmill running is associated with a shorter unsupported (flight) phase, decreased step length and increased cadence (number of steps / min) [16-17], and with a more flat foot positioning at landing [18] compared to over-ground running. Additionally, treadmill running is characterized by decreased ground reaction forces (GRF) components (peak propulsive force and peak medial force), associated with the reduced knee moments recorded. Nevertheless, the higher ankle moments and preserved power recorded support the preservation of push-off during treadmill running [16]. As ground reaction forces are decreased while running on a treadmill, it could be expected some muscles to require less intensities of activation since metabolic cost is reduced [19-20]. According to Hunter et al. [21] findings, most of the lower limb muscles showed decreases in activation as more body weight was supported, while running on a positive-pressure treadmill. Specifically, the two vastii muscles (medialis and lateralis) and rectus femoris activities decreased dramatically as more body weight was supported.
Currently, most of the research has examined the effect of varied slope gradients and running environments on the kinematic and kinetic characteristics of running, while little information is available on the influence of these variables on the lower limb muscle activation patterns. The purpose of this study was to assess whether the electromyographic (EMG) activity of the lower limb muscles during running is affected by a) different running environments (over-ground and treadmill running) and b) varied slope gradients (downhill and uphill running). It was hypothesized that the EMG activity of the lower limb muscles would be higher when over-ground running than treadmill running. Additionally, it was expected that the different slope gradients (negative and positive gradients) would affect muscle activity compared to level running.

\section{Methods}

\subsection{Participants}

Ten healthy male recreational runners (age: $21 \pm 2.5$ years, height: $1.75 \pm 10.5 \mathrm{~cm}$, body mass: $69.1 \pm 5.7 \mathrm{~kg}$ ) participated in the current study. All participants were physically active, free from injuries and able to run $10 \mathrm{~km}$ in $55-60 \mathrm{~min}$ at inclined surfaces and level terrain. After receiving detailed information on the objectives and the procedures of the study, each participant signed an informed consent form.

\subsection{Procedures and Materials}

Each of the ten participants, followed a standardized 10 min warm-up and then was assessed in two different running conditions, treadmill running and over-ground running, in a randomized order. Each running condition consisted of a $20 \mathrm{~m}$ distance running at a velocity of $11 \pm 1 \mathrm{~km} / \mathrm{h}$ at each of the following slope gradients, $-8 \%,-4 \%$, $0 \%, 4 \%, 8 \%$, in a randomized order. The velocity of $11 \pm 1 \mathrm{~km} / \mathrm{h}$ was chosen as it is a common velocity of training for many of the long runs recreational runners complete. Thus, each participant performed a total of $5 \times 20 \mathrm{~m}$ trials, with a 5 min interval, in each running condition. A 24 hour rest intervened between each condition.

For the treadmill running condition, a Pegasus (UK) T1600 training treadmill was used. In over-ground running condition, slope gradients were measured with a gradiometer GemRed (China) Digital Protractor.

\subsection{Variables}

Running environment and slope gradient were the independent variables. Running environment was determined by over-ground and treadmill running. Slope gradient was defined by five different inclinations: $-8 \%,-4 \%, 0 \%, 4 \%$, $8 \%$.

The electromyographic activity measured in $\mu \mathrm{V}$ of the following four muscles of the right lower limb - rectus femoris, vastus medialis, bicep femoris and gastrocnemius was the dependent variable. 


\subsection{EMG Recording and Processing}

The electromyographic activity of the lower limb muscles was recorded using an EMG wireless device (Delsys (USA) Wireless Myomonitor Trigger, Module DS-U05). After the skin surface was shaved and cleaned, rectangular-shaped $(19.8 \mathrm{~mm} \times 35 \mathrm{~mm})$ bipolar surface electrodes $(1 \mathrm{~cm} \mathrm{Ag}$ conductors with an inter-electrode distance of $1 \mathrm{~cm}$ ) were used to acquire the EMG activity from the following four muscles of the right lower limb: rectus femoris, vastus medialis, bicep femoris and gastrocnemius. Electrodes were placed on the midpoint of the contracted muscle along the longitudinal axis, based on SENIAM project recommendations for surface EMG electrode placement. For data recording and processing an EMG data recorder at $1024 \mathrm{~Hz}$ was used (EMG works 3.7 Delsys Inc.). EMG activity was recorded for the whole $20 \mathrm{~m}$ running performance. All four muscles were recorded simultaneously.

\subsection{Statistical Analysis}

Descriptive statistics were used in order to calculate the mean and SD of the electromyographic activity of the lower limb muscles. Test of normal distribution (Shapiro - Wilks test) was also conducted. A two-way repeated measures ANOVA [2(treadmill running, over-ground running) $x$ 5(slope gradients)] was performed in order to assess the effect of the running condition and the slope gradients on the EMG activity of the four muscle groups. A three-way ANOVA model (running condition x slope gradient $\mathrm{x}$ muscle group) was performed in order to assess the difference in lower limb muscle activation between over-ground and treadmill running. Additionally, a two-way ANOVA model (slope gradient $\mathrm{x}$ muscle group) was performed in order to assess likely differences in muscle activation between slope gradients, when over-ground and treadmill running. Post-hoc analysis was performed using the Bonferroni test. The significance level was set at $\mathrm{p}<0.05$. The data were analysed with SPSS version 17.00 for windows (SPSS Inc, Chicago, IL).

\section{Results}

Mean and SD of EMG activity of the lower limb muscles, when over-ground and treadmill running, at each slope gradient are presented in Table 1. Assessing EMG activity of the bicep femoris muscle, Repeated ANOVA's results revealed a significant interaction "running condition $\mathrm{x}$ slope gradient" effect $(\mathrm{F}(1,9)=15.02, \mathrm{p}=0.004)$. A significantly greater activation of the bicep femoris was observed when over-ground running, at all slope gradients, compared to treadmill running. However, at the higher gradient $(8 \%)$ this difference was not statistically significant.

During treadmill running, a significant effect of the factor "slope gradient" was observed on bicep femoris EMG activity. At more pronounced gradients (-8\% and $8 \%)$, bicep femoris activity was significantly higher compared to less pronounced gradients and level running. Additionally, with a positive increase in slope gradient (from $-4 \%$ to $8 \%$ ) muscle activity significantly increased. On the contrary, when participants run over-ground, results revealed that the different slope gradients did not significantly influence muscle activity.

Repeated ANOVA's results revealed a significant interaction "running condition x slope gradient" effect $(\mathrm{F}(1,9)$ $=53.34, \mathrm{p}<0.0001)$ on the gastrocnemius EMG activity. Comparing the two running conditions, it seems that gastrocnemius was significantly more activated when overground running, at slope gradients of $-4 \%, 0 \%, 4 \%$. At more pronounced gradients $( \pm 8 \%)$, there were not significant differences in muscle activation between the two running conditions.

When participants run on the treadmill, the slope gradient had a significant effect on gastrocnemius EMG activity. At more pronounced gradients $( \pm 8 \%)$, muscle activity was significantly higher compared to level running, while at the gradient of $-4 \%$, a significantly smaller muscle activity was observed. Additionally, a pattern of increased activation was observed with a positive increase in slope gradients. When participants run over-ground, at the gradient of $4 \%$, a significantly lower gastrocnemius activity was observed compared to all the other slope gradients.

Assessing Rectus femoris EMG activity, Repeated ANOVA's results showed a significant interaction "running condition $x$ slope gradient" effect $(F(1,9)=76.25$, $p<0.0001)$. Comparing the two running conditions, a significantly higher activation of the Rectus femoris was observed when overground running, at all slope gradients.

The slope gradient had a significant effect on Rectus femoris EMG activity, when treadmill running. At more pronounced gradients $( \pm 8 \%)$, muscle activity was significantly higher compared to lower gradients $( \pm 4 \%)$ and level running. Interestingly, a significantly higher muscle activity was also observed when level running compared to hill running at gradients $\pm 4 \%$. When running over-ground, at pronounced gradients $( \pm 8 \%)$, a similar pattern of Rectus femoris EMG activity was observed. Additionally, with a positive increase in slope gradient (from $-4 \%$ to $4 \%$ ) Rectus femoris activity was significantly increased.

Assessing Vastus medialis EMG activity, Repeated ANOVA's results showed a significant interaction "running condition $x$ slope gradient" effect $(F(1,9)=45.93$, $p<0.0001)$. A significantly higher activation of the vastus medialis was observed when running over-ground, at all slope gradients except the gradient of $-8 \%$, compared to treadmill running.

The slope gradient had a significant effect on vastus medialis EMG activity, when participants run on a treadmill. At more pronounced gradients $( \pm 8 \%)$, muscle activity was significantly greater compared to lower gradients $( \pm 4 \%)$ and level running, while at the gradient of $-4 \%$ a significantly smaller EMG activity was observed. When over-ground running, with a positive increase in slope gradient (from $-4 \%$ to $8 \%$ ) vastus medialis activity significantly increased. Although at $-8 \%$ gradient muscle activity was significantly higher compared to $-4 \%$ and level running, this value was 
almost the same with the value of EMG activity recorded at $4 \%$ gradient.

Table 1. Mean and SD of EMG activity of the lower limb muscles, when over-ground and treadmill running at each slope gradient.

\begin{tabular}{|c|c|c|c|c|c|c|c|c|}
\hline \multirow{2}{*}{$\begin{array}{l}\text { Slope } \\
\text { gradient }\end{array}$} & \multicolumn{4}{|c|}{ Over ground running } & \multicolumn{4}{|c|}{ Treadmill running } \\
\hline & $\begin{array}{l}\text { Bicep } \\
\text { femoris }\end{array}$ & $\begin{array}{l}\text { Gastro } \\
\text { cnemius }\end{array}$ & $\begin{array}{l}\text { Rectus } \\
\text { femoris }\end{array}$ & $\begin{array}{l}\text { Vastus } \\
\text { medialis }\end{array}$ & Bicep femoris & $\begin{array}{l}\text { Gastro } \\
\text { cnemius }\end{array}$ & $\begin{array}{l}\text { Rectus } \\
\text { femoris }\end{array}$ & $\begin{array}{l}\text { Vastus } \\
\text { medialis }\end{array}$ \\
\hline$-8^{\circ}$ & $78.91 * \pm 7.93$ & $91.61^{\mathrm{e}} \pm 6.04$ & $71.57^{* \mathrm{~b}, \mathrm{~d}, \mathrm{f}} \pm 7.81$ & $55.07^{\mathrm{b}, \mathrm{d}, \mathrm{h}} \pm 6.43$ & $73.69^{\mathrm{b}, \mathrm{d}, \mathrm{h}} \pm 4.17$ & $89.72^{\mathrm{b}, \mathrm{d}, \mathrm{f}, \mathrm{h}} \pm 6.95$ & $62.80^{\mathrm{b}, \mathrm{d}, \mathrm{f}, \mathrm{h}} \pm 8.41$ & $54.11^{\mathrm{b}, \mathrm{d}, \mathrm{f}} \pm 7.13$ \\
\hline$-4^{\circ}$ & $75.37 * \pm 6.67$ & $90.64^{* \mathrm{f}} \pm 6.85$ & $55.48^{* \mathrm{~d}, \mathrm{f}, \mathrm{h}} \pm 6.69$ & $45.07^{* \mathrm{~d}, \mathrm{f}, \mathrm{h}} \pm 6.09$ & $61.98^{\mathrm{d}, \mathrm{ffh}} \pm 6.43$ & $57.15^{\mathrm{d}, \mathrm{f,h}} \pm 7.41$ & $41.42^{\mathrm{d}, \mathrm{h}} \pm 7.01$ & $36.30^{\mathrm{d}, \mathrm{f}, \mathrm{h}} \pm 7.14$ \\
\hline $0^{\circ}$ & $78.49 * \pm 8.41$ & $92.84 *{ }^{\mathrm{f}} \pm 7.31$ & $62.56^{* \mathrm{e}, \mathrm{h}} \pm 9.74$ & $49.95^{* \mathrm{f}, \mathrm{h}} \pm 6.08$ & $66.15^{\mathrm{e}, \mathrm{h}} \pm 6.26$ & $75.36^{\mathrm{h}} \pm 6.93$ & $48.79^{\mathrm{f}, \mathrm{h}} \pm 6.59$ & $39.45^{\mathrm{h}} \pm 6.65$ \\
\hline $4^{\circ}$ & $81.20 * \pm 9.34$ & $86.70^{* \mathrm{~h}} \pm 6.51$ & $66.19 * \mathrm{~h} \pm 7.29$ & $54.40^{* \mathrm{~h}} \pm 5.81$ & $69.66^{\mathrm{h}} \pm 8.26$ & $75.68^{\mathrm{h}} \pm 5.88$ & $43.00^{\mathrm{h}} \pm 7.87$ & $40.99^{\mathrm{h}} \pm 7.79$ \\
\hline $8^{\circ}$ & $79.59 \pm 16.08$ & $93.21 \pm 8.06$ & $73.08 * \pm 7.00$ & $59.40 * \pm 7.53$ & $83.32 \pm 5.63$ & $93.20 \pm 8.00$ & $67.34 \pm 7.24$ & $56.72 \pm 8.67$ \\
\hline
\end{tabular}

* Significant differences in muscle activation $(\mathrm{p}<0.01)$ between over-ground and treadmill running

Significant differences between slope gradient: a: $\mathrm{p}<0.05$ vs $-4^{\circ}$, c: $\mathrm{p}<0.05$ vs $0^{\circ}$, e: $\mathrm{p}<0.05$ vs $4^{\circ}$, g: $<<0.05$ vs $8^{\circ}$, b: $p<0.005$ vs $-4^{\circ}, \mathrm{d}: \mathrm{p}<0.005$ vs $0^{\circ}, \mathrm{f}$ : $\mathrm{p}<0.005$ vs $4^{\circ}, \mathrm{h}: \mathrm{p}<0.005$ vs $8^{\circ}$

Overall, EMG activity of the lower limb muscles was $16.54 \% \quad(\mathrm{p}<0.0001)$ higher when over-ground running compared to treadmill running (Table 2).

Table 2. Differences in lower limb muscle activation between over-ground and treadmill running, independent of slope gradient and muscle group.

\begin{tabular}{llll}
\hline $\begin{array}{l}\text { Running } \\
\text { condition }\end{array}$ & Mean \pm SD & $\begin{array}{l}\text { Mean diff } \\
(\mathbf{9 5 \%} \text { CI) }\end{array}$ & $\begin{array}{l}\% \\
\text { difference }\end{array}$ \\
\hline $\begin{array}{l}\text { Over-ground } \\
\text { Treadmill }\end{array}$ & $\begin{array}{l}72.07 * \pm 1.2 \\
61.84 \pm 1.1\end{array}$ & $10.22(9.41-11.04)$ & $16.54 \%$ \\
\hline
\end{tabular}

* Significant difference in lower limb muscle activation $(\mathrm{p}<0.0001)$

between over-ground and treadmill running

Assessing the effect of the slope gradient on muscle activity, it seems that uphill running is associated with significantly greater activation compared to level and downhill running, for both running conditions. Downhill running caused significantly greater muscle activation compared to level running, only on the treadmill condition (Table 3).

Table 3. Differences in lower limb muscle activation between slope gradients for over-ground and treadmill running, independent of muscle group.

\begin{tabular}{lll}
\hline Slope gradient & Over-ground running & Treadmill running \\
\hline Downhill & $70.46 \pm 1.1$ & $59.64^{\mathrm{e}} \pm 1.1$ \\
Level & $70.96 \pm 1.3$ & $57.44 \pm 1.1$ \\
Uphill & $74.22^{\mathrm{a}, \mathrm{b}} \pm 1.4$ & $66.24^{\mathrm{c}, \mathrm{d}} \pm 1.2$ \\
\hline
\end{tabular}

Significant differences between slope gradients:

$\mathrm{a}: \mathrm{p}<0.0001$ vs level running

b: $p<0.0001$ vs downhill running

c: $p<0.0001$ vs level running

$\mathrm{d}: \mathrm{p}<0.0001$ vs downhill running

e: $p<0.0001$ vs level running

\section{Discussion}

The purpose of the present study was to assess EMG activity of the lower limb muscles during running at different environments (over-ground $v s$ treadmill) and at varied slope gradients $(-8 \%,-4 \%, 0 \%, 4 \%, 8 \%)$. Assessing each muscle group separately, it was found that all four muscles' activation was significantly higher during over-ground running compared to treadmill running, confirming the first hypothesis of our study. During over-ground running, EMG activity of the lower limb muscles was $16.54 \%(p<0.0001)$ higher compared to treadmill running. When running on a treadmill, the supporting ground (treadmill belt) is moving relatively to subjects' centre of mass [15], implying locomotion with less effort. According to literature, differences in kinematic and kinetic parameters have been observed between over-ground and treadmill running. Treadmill running is characterized by a shorter flight phase of the gait cycle, decreased step length and increased step frequency compared to over-ground running [16-18]. The swing amplitude of the leg, the vertical displacement and the variance in vertical and horizontal velocity were lower in treadmill running [17]. These differences in spatiotemporal gait characteristics could be associated with the work performed by muscle groups. When running on a treadmill decreased ground reaction forces have been observed [16] which is associated with a reduced metabolic cost, consequently with lower muscle activation requirements [20, 21].

Running at different slope gradients seems to influence lower limb muscle activation. Results revealed that when uphill running, lower limb muscle activation was significantly higher compared to level and downhill running, for both running conditions (over-ground and treadmill running). Increased muscle activation with a positive increase in slope gradient may be attributed to a greater mechanical load on the lower limb muscles when uphill running [7]. It has been reported that uphill running is characterized by increased demands for muscle function in order to increase the body potential energy [8]. Running at inclined surfaces (30\% grade) was related with changes in segmental and muscular coordination compared to level running. Higher step frequency and a greater proportion of the gait cycle spent in stance were observed while incline running. These differences in kinetic and kinematic characteristics were accompanied by increases in the activity of specific muscles. Specifically, gluteus maximus, vastus lateralis, soleus, rectus femoris and gastrocnemius found to be more active during incline running compared to level running [7]. Additionally, physiological parameters such as oxygen consumption, heart rate and blood lactate concentration were greater during uphill running compared to level running [22-24]. According to the current findings, rectus femoris and vastus medialis activity significantly increased with a positive increase in slope gradient (from $-4 \%$ to $8 \%$ ). Gastrocnemius muscle 
presented its greater EMG activation at the higher gradient $(8 \%)$. These findings are in accordance with the existing literature. During running at a slope gradient of $9.1 \%$ the vastus and rectus femoris muscles found to be more active compared to level running [9]. Additionally, an increased muscle activity in knee extensors was reported with increasing running incline $[25,26]$.

Additionally, the findings of the present study revealed that when running on a treadmill the negative slope gradient (downhill running) was associated with significantly greater muscle activation compared to level running, while when over-ground running, EMG activity presented no significant differences between downhill and level running. Downhill running is characterized by greater knee flexion angle which is associated with an increased vertical displacement of the centre of mass and an increased maximal vertical force value [3]. Higher gastrocnemius and bicep femoris activity have been observed when downhill running, while adopting anterior foot strike patterns [10-12, 14]. Although the foot strike pattern was not assessed in the current study, gastrocnemius and bicep femoris muscles also presented greater EMG activity when downhill running at the slope of $8 \%$ compared to level running.

During downhill or uphill running at severe slopes $( \pm 8 \%)$, rectus femoris and vastus medialis activity were significantly higher compared to lower gradients and level running, in both running conditions (over-ground and treadmill running). However, a similar pattern of activation at more pronounced slopes for bicep femoris and gastrocnemius was observed only when treadmill running. It is suggested that increasing gradients may alter spatiotemporal gait characteristics [3-5, 7], and might impair athletes' ability to use efficiently ground reaction forces to increase step length and flight time in a shorter stretch - shortening cycle [27]. Padulo et al. [27] suggest that athletes have to apply greater forces to maintain running velocity with severe gradients, which may be associated with higher muscle activation.

\section{Conclusion}

The results of the present study revealed that over-ground running caused a significantly higher EMG activity (16.54\%) of the lower limb muscles compared to treadmill running. Additionally, varied slope gradients found to affect muscle activation. During uphill running, lower limb muscle activation was significantly higher compared to level and downhill running, independently of running environment. However, running at negative slope gradients was associated with significantly greater muscle activation compared to level running, only on the treadmill condition. The above findings should be further examined in relation to spatiotemporal adaptations, foot placement patterns and ground reaction forces required during running at different slope gradients. Data from our study could be useful for the design of special training protocols for different style runners.

\section{References}

[1] Lussiana T, Fabre N, Hebert-Losier K, et al. Effect of slope and footwear on running economy and kinematics. Scand J Med Sci Sports 2013; 23: 246-253.

[2] Farley CT, Houdijk HP, Van Strien C, et al. Mechanism of leg stiffness adjustment for hoping on surfaces of different stiffness. J Appl Physiol 1998; 85: 1044-1055.

[3] Lussiana T, Hebert-Losier K, Mourot L. Effect of minimal shoes and slope on vertical and leg stiffness during running. J Sport Health Sci 2015; 4: 195-202.

[4] Garcia-Pinillos F, Latorre-Roman PA, Ramirez-Campillo R, et al. How does the slope gradient affect spatiotemporal parameters during running? Influence of athletic level and vertical and leg stifness. Gait Posture 2018; 68: 72-77.

[5] Gottschall JS, Kram R. Ground reaction forces during downhill and uphill running. J Biomech 2005; 38: 445-452.

[6] Telhan G, Franz JR, Dicharry J, et al. Lower limb joint kinetics during moderately sloped running. J Athl Train 2010; 45: 16-21.

[7] Swanson SC, Caldwell GE. An integration biomechanical analysis of high speed incline and level treadmill running. Med Sci Sport Exerc 2000; 32: 1146-1155.

[8] Roberts TJ, Belliveau RA. Sources of mechanical power for uphill running in humans. J Exp Biol 2005; 208: 1963-1970.

[9] Yokozawa T, Fujii N, Ae M. Muscle activities of the lower limb during level and uphill running. $\mathrm{J}$ Biomech 2007; 40: 3467-3475.

[10] Ahn AN, Brayton C, Bhatia T, et al. Muscle activity and kinematics of forefoot and rearfoot strike runners. J Sport Health Sci 2014; 3: 102-112.

[11] Giandolini M, Arnal PJ, Millet GY, et al. Impact reduction during running: efficiency of simple acute interventions in recreational runners. Eur J Appl Physiol 2013; 113: 599-609.

[12] Yong JR, Silder A, Delp SL. Differences in muscle activity between natural forefoot and rearfoot strikers during running. J Biomech 2014; 47: 3593-3597.

[13] Shih Y, Lin KL, Shiang TY. Is the foot striking pattern more important than barefoot or shod conditions in running? Gait Posture 2013; 38: 490-494.

[14] Warne JP, Warrington GD. Four-week habituation to simulated barefoot running improves running economy when compared with shod running. Scand J Med Sci Sports 2012; 24: 563-568.

[15] Morin JB, Seve P. Sprint running performance: comparison between treadmill and field conditions. Eur J Appl Physiol 2011; 111: 1695-1703.

[16] Riley PO, Dicharry J, Franz J, et al. A kinematic and kinetic comparison of over-ground and treadmill running. Med Sci Sport Exerc 2008; 40:1093-1100.

[17] Wank V, Frick U, Schmidtbleicher, D. Kinematics and electromyography of lower limb muscles in over-ground and treadmill running. Int J Sports Med 1998; 19: 455-461. 
[18] Nigg BM, DeBoer RW, Fischer V. A kinematic comparison of over-ground and treadmill running. Med Sci Sport Exerc 1995; 27: 98-105.

[19] Grabowski AM. Metabolic and biomechanical effects of velocity and weight support using a lower body positive pressure device during walking. Arch Phys Med Rehab 2010; 91: 951-957.

[20] Grabowski AM, Kram R. Effects of velocity and weight support on ground reaction forces and metabolic power during running. J Appl Biomech 2008; 24: 288-297.

[21] Hunter I, Seeley MK, Hopkins JT, et al. EMG activity during positive - pressure treadmill running. J Electromyogr Kinesiol 2014; 24: 348-352.

[22] Gregor RJ, Costill DL. A comparison of the energy expenditure during positive and negative grade running. $\mathrm{J}$ Sports Med 1973; 13: 248-252.
[23] Pivarnik JM, Sherman NW. Responses of aerobically fit men and women to uphill / downhill walking and slow jogging. Med Sci Sport Exerc 1990; 22: 127-130.

[24] Staab JS, Agnew JW, Siconolfi SF. Metabolic and performance responses to uphill and downhill running in distance runners. Med Sci Sport Exerc 1992; 24: 124-127.

[25] Sloniger MA, Cureton KJ, Prior BM, et al. Anaerobic capacity and muscle activation during horizontal and uphill running. J Appl Physiol 1997a; 83: 262-269.

[26] Sloniger MA, Cureton KJ, Prior BM, et al. Lower extremity muscle activation during horizontal and uphill running. J Appl Physiol 1997b; 83: 2073-2079.

[27] Padulo J, Annino G, Migliaccio GM, et al. Kinematics of running at different slopes and speeds. J Strength Cond Res 2012; 26: 1331-1339. 